\title{
On the Refined Hermite-Hadamard Inequalities
}

\author{
Tahir Ali*, Muhammad Adil Khan, Adem Kilicman and Qamar Din
}

(Communicated by Nihal YILMAZ ÖZGÜR)

\begin{abstract}
In this paper, we give some new refinements of Hermite-Hadamard inequality for co-ordinated convex function. These refinements provide us better estimation as compare to the earlier established refinements of Hadamard's inequality.
\end{abstract}

Keywords: Convex function; Co-ordinated convex function; Hermite-Hadamard's inequality.

AMS Subject Classification (2010): Primary: 26D15; Secondary: 26A33; $26 D 10$.

${ }^{*}$ Corresponding Author

\section{Introduction}

Let $I$ be an interval in $\mathbb{R}$ and $f: I \subseteq \mathbb{R} \rightarrow \mathbb{R}$ such that $a, b \in \mathrm{I}$ with $a<b$. Then the following double inequality:

$$
f\left(\frac{a+b}{2}\right) \leq \frac{1}{b-a} \int_{a}^{b} f(x) d x \leq \frac{f(a)+f(b)}{2}
$$

is well known in the literature as Hadamard's inequality for convex mappings. Note that some of the classical inequalities for means can be derived from Hadamard's for appropriate particular selections of the mapping $f$. The inequality $(1.1)$ which appeared for the first time in $([11], 1893)$ gives us an estimate of the mean value of a convex function $f$. Since then, many important refinements of Hermite-Hadamard inequality for convex functions have been investigated extensively. For example see [1]-[18].

In ([6], 1992) S. S. Dragomir defined a mapping $\mathrm{H}$ in the following way:

$$
\mathrm{H}(t)=\frac{1}{b-a} \int_{a}^{b} f\left(t x+(1-t) \frac{a+b}{2}\right) d x
$$

where $\mathrm{H}:[0,1] \rightarrow \mathbb{R}$ and $f:[a, b] \rightarrow \mathbb{R}$ is a convex function defined on $[a, b]$, and proved a new refinement of Hadamard's inequality:

Theorem 1.1 ([6]). Let $f:[a, b] \rightarrow \mathbb{R}$ be a convex function and $H:[0,1] \rightarrow \mathbb{R}$ be defined as above, then we have:

$$
f\left(\frac{a+b}{2}\right) \leq H(t) \leq \frac{1}{b-a} \int_{a}^{b} f(x) d x
$$

In ([3], 2010), A. E. Farissi established a simple proof and a new generalization of the inequality (1.1) as follows:

Theorem $1.2([3])$. Assume that $f:[a, b] \rightarrow \mathbb{R}$ is a convex function on $[a, b]$, then for all $t \in[0,1]$ we have:

$$
f\left(\frac{a+b}{2}\right) \leq I(t) \leq \frac{1}{b-a} \int_{a}^{b} f(x) d x \leq L(t) \leq \frac{f(a)+f(b)}{2},
$$

Received : 17-03-2016, Accepted : 01-02-2018 
where

$$
I(t)=: t f\left(\frac{t b+(2-t) a}{2}\right)+(1-t) f\left(\frac{(1+t) b+(1-t) a}{2}\right)
$$

and

$$
L(t)=: \frac{1}{2}[f(t b+(1-t) a)+t f(a)+(1-t) f(b)] .
$$

To complete this section we state definition of co-ordinated convex function and its related Hermite-Hadamard type inequalities:

Let us consider the bi-dimensional interval $\Delta:=[a, b] \times[c, d]$ in $\mathbb{R}^{2}$ with $a<b$ and $c<d$, a mapping $f: \Delta \rightarrow \mathbb{R}$ is said to be convex on $\Delta$ if the inequality

$$
f(\lambda x+(1-\lambda) z, \lambda y+(1-\lambda) w) \leq \lambda f(x, y)+(1-\lambda) f(z, w)
$$

holds for all $(x, y),(z, w) \in \Delta$ and $\lambda \in[0,1]$.

A function $f: \Delta \rightarrow \mathbb{R}$ is said to be convex on the co-ordinates on $\Delta$ if the partial mappings $f_{y}:[a, b] \rightarrow \mathbb{R}$, $f_{y}(u)=f(u, y)$ and $f_{x}:[c, d] \rightarrow \mathbb{R}, f_{x}(v)=f(x, v)$ are convex for all $y \in[c, d]$ and $x \in[a, b]$.

A formal definition for co-ordinated convex functions may be stated as follows:

Definition 1.1. A function $f: \Delta \rightarrow \mathbb{R}$ is said to be convex on co-ordinates on $\Delta$ if the inequality

$$
\begin{aligned}
f(\lambda x+(1-\lambda) z, t y+(1-t) w) & \leq \lambda t f(x, y)+\lambda(1-t) f(x, w) \\
& +(1-\lambda) t f(z, y)+(1-t)(1-\lambda) f(z, w)
\end{aligned}
$$

holds for all $(x, y),(x, w),(z, y),(z, w) \in \Delta$ and $t \in[0,1], \lambda \in[0,1]$.

S. S. Dragomir in [7] established the following Hadamard-type inequalities for co-ordinated convex functions:

Theorem 1.3 ([7]). Suppose that $f:[a, b] \times[c, d] \rightarrow \mathbb{R}$ is convex on the co-ordinates on $\Delta$. Then one has the inequalities:

$$
\begin{aligned}
& f\left(\frac{a+b}{2}, \frac{c+d}{2}\right) \\
\leq & \frac{1}{2}\left[\frac{1}{b-a} \int_{a}^{b} f\left(x, \frac{c+d}{2}\right) d x+\frac{1}{d-c} \int_{c}^{d} f\left(\frac{a+b}{2}, y\right) d y\right] \\
\leq & \frac{1}{(b-a)(d-c)} \int_{a}^{b} \int_{c}^{d} f(x, y) d x d y \\
\leq & \frac{1}{4}\left[\frac{1}{b-a} \int_{a}^{b} f(x, c) d x+\frac{1}{b-a} \int_{a}^{b} f(x, d) d x\right. \\
+ & \left.\frac{1}{d-c} \int_{c}^{d} f(a, y) d y+\frac{1}{d-c} \int_{c}^{d} f(b, y) d y\right] \\
\leq & \frac{f(a, c)+f(a, d)+f(b, c)+f(b, d)}{4} .
\end{aligned}
$$

The above inequalities are sharp.

In ([1], 2008), M. Alomari and Darus defined co-ordinated s-convex functions and proved some inequalities. In ([16], 2009), analogous results for h-convex functions on the co-ordinates were proved by M. A. Latif and M. Alomari.

In ([2], 2009), Alomari et al. established some Hadamard type inequalities for co-ordinated log-convex functions. In ([17]), M. A. Latif and S. S. Dragomir obtained some new Hadamard type inequalities for differentiable coordinated convex and concave functions.

For recent results and generalizations concerning Hermite-Hadamard type inequality for co-ordinated convex functions see ([18], 2012) and some of the references given therein.

In this paper, we obtain some new Hermite-Hadamard type inequalities for convex functions on the co-ordinates. These results refine the Hermite-Hadamard type inequalities given in Theorem 1.3 as well as in [3]. 


\section{Refinements}

Now, we give first refinement of Hermite-Hadamard inequality for co-ordinated convex function.

Theorem 2.1. Suppose that $f:[a, b] \times[c, d] \rightarrow \mathbb{R}$ is convex on the co-ordinates on $\Delta$, then we have the following refinement:

$$
\begin{aligned}
& f\left(\frac{a+b}{2}, \frac{c+d}{2}\right) \\
\leq & \Omega_{1}(t, s) \\
\leq & \frac{1}{2}\left[\frac{1}{b-a} \int_{a}^{b} f\left(x, \frac{c+d}{2}\right) d x+\frac{1}{d-c} \int_{c}^{d} f\left(\frac{a+b}{2}, y\right) d y\right] \\
\leq & \Omega_{2}(t, s) \\
\leq & \frac{1}{(b-a)(d-c)} \int_{a}^{b} \int_{c}^{d} f(x, y) d y d x
\end{aligned}
$$

where

$$
\Omega_{1}(t, s)=\frac{1}{2}\left[\frac{1}{b-a} \int_{a}^{b} f\left(t x+(1-t) \frac{a+b}{2}, \frac{c+d}{2}\right) d x+\frac{1}{d-c} \int_{c}^{d} f\left(\frac{a+b}{2}, s y+(1-s) \frac{c+d}{2}\right) d y\right]
$$

and

$$
\Omega_{2}(t, s)=\frac{1}{2}\left[\frac{1}{(b-a)(d-c)} \int_{a}^{b} \int_{c}^{d}\left[f\left(x, s y+(1-s) \frac{c+d}{2}\right)+f\left(t x+(1-t) \frac{a+b}{2}, y\right)\right] d y d x\right] .
$$

Proof. Since $f: \Delta=[a, b] \times[c, d] \rightarrow \mathbb{R}$ is convex on the co-ordinates on $\Delta$, it follows that the mapping $f_{x}:[c, d] \rightarrow \mathbb{R}$, $f_{x}(v)=f(x, v)$ is convex on $[c, d]$ for all $x \in[a, b]$. Then by making use of Theorem 1.2 one has

$$
f_{x}\left(\frac{c+d}{2}\right) \leq \frac{1}{d-c} \int_{c}^{d} f_{x}\left(s y+(1-s) \frac{c+d}{2}\right) d y \leq \frac{1}{d-c} \int_{c}^{d} f_{x}(y) d y .
$$

Integrating this inequality on $[a, b]$, we have

$$
\begin{aligned}
& \frac{1}{b-a} \int_{a}^{b} f\left(x, \frac{c+d}{2}\right) d x \\
\leq & \frac{1}{(b-a)(d-c)} \int_{a}^{b} \int_{c}^{d} f\left(x, s y+(1-s) \frac{c+d}{2}\right) d y d x \\
\leq & \frac{1}{(b-a)(d-c)} \int_{a}^{b} \int_{c}^{d} f(x, y) d y d x .
\end{aligned}
$$

By a similar argument applied for the mapping $f_{y}:[a, b] \rightarrow \mathbb{R}, f_{y}(u)=f(u, y)$ is convex on $[a, b]$ for all $y \in[a, b]$, we get

$$
\begin{aligned}
& \frac{1}{d-c} \int_{c}^{d} f\left(\frac{a+b}{2}, y\right) d y \\
\leq & \frac{1}{(b-a)(d-c)} \int_{a}^{b} \int_{c}^{d} f\left(t x+(1-t) \frac{a+b}{2}, y\right) d y d x \\
\leq & \frac{1}{(b-a)(d-c)} \int_{a}^{b} \int_{c}^{d} f(x, y) d y d x .
\end{aligned}
$$

Summing the inequalities (2.5) and (2.6), we get the third and the fourth inequalities in (2.1). By Hadamard's inequality, we also have

$$
f\left(\frac{a+b}{2}, \frac{c+d}{2}\right) \leq \frac{1}{b-a} \int_{a}^{b} f\left(t x+(1-t) \frac{a+b}{2}, \frac{c+d}{2}\right) d x \leq \frac{1}{b-a} \int_{a}^{b} f\left(x, \frac{c+d}{2}\right) d x
$$


and

$$
f\left(\frac{a+b}{2}, \frac{c+d}{2}\right) \leq \frac{1}{d-c} \int_{c}^{d} f\left(\frac{a+b}{2}, s y+(1-s) \frac{c+d}{2}\right) d y \leq \frac{1}{d-c} \int_{c}^{d} f\left(\frac{a+b}{2}, y\right) d y
$$

which give, by addition, the first and the second inequalities in (2.1).

Theorem 2.2. Suppose that $f: \Delta=[a, b] \times[c, d] \rightarrow \mathbb{R}$ is co-ordinated convex on $\Delta$. Then for all $t \in[0,1], s \in[0,1]$, one has the inequalities:

$$
\begin{aligned}
f\left(\frac{a+b}{2}, \frac{c+d}{2}\right) & \leq \text { A } \\
& \leq \gamma(t, s) \\
& \leq \frac{1}{(b-a)(d-c)} \int_{a}^{b} \int_{c}^{d} f(x, y) d y d x \\
& \leq \Gamma(t, s) \\
& \leq B \\
& \leq \frac{f(a, c)+f(a, d)+f(b, c)+f(b, d)}{4}
\end{aligned}
$$

where

$$
\begin{aligned}
A= & \frac{1}{2}\left[\frac{1}{b-a} \int_{a}^{b} f\left(x, \frac{c+d}{2}\right) d x+\frac{1}{d-c} \int_{c}^{d} f\left(\frac{a+b}{2}, y\right) d y\right] \\
B= & \frac{1}{4}\left[\frac{1}{b-a} \int_{a}^{b} f(x, c) d x+\frac{1}{b-a} \int_{a}^{b} f(x, d) d x+\frac{1}{d-c} \int_{c}^{d} f(a, y) d y+\frac{1}{d-c} \int_{c}^{d} f(b, y) d y\right] \\
\gamma(t, s)= & \frac{1}{2}\left[\frac{1}{b-a} \int_{a}^{b}\left[s f\left(x, \frac{s d+(2-s) c}{2}\right)+(1-s) f\left(x, \frac{(1+s) d+(1-s) c}{2}\right)\right] d x\right. \\
& \left.+\frac{1}{d-c} \int_{c}^{d}\left[t f\left(\frac{t b+(2-t) a}{2}, y\right)+(1-t) f\left(\frac{(1+t) b+(1-t) a}{2}, y\right)\right] d y\right]
\end{aligned}
$$

and

$$
\begin{aligned}
\Gamma(t, s) & =\frac{1}{4}\left[\frac{1}{b-a} \int_{a}^{b}[f(x, s d+(1-s) c)+s f(x, c)+(1-s) f(x, d)] d x\right. \\
& \left.+\frac{1}{d-c} \int_{c}^{d}[f(t b+(1-t) a, y)+t f(a, y)+(1-t) f(b, y)] d y\right]
\end{aligned}
$$

Proof. Since $f: \Delta=[a, b] \times[c, d] \rightarrow \mathbb{R}$ is convex on the co-ordinates on $\Delta$, it follows that the mapping $f_{x}:[c, d] \rightarrow \mathbb{R}$, $f_{x}(v)=f(x, v)$ is convex on $[c, d]$ for all $x \in[a, b]$. Then under the utility of Theorem 1.2 one has

$$
f_{x}\left(\frac{c+d}{2}\right) \leq s f_{x}\left(\frac{s d+(2-s) c}{2}\right)+(1-s) f_{x}\left(\frac{(1+s) d+(1-s) c}{2}\right) \leq \frac{1}{d-c} \int_{c}^{d} f_{x}(y) d y
$$

and integrating this inequality over $[a, b]$, we have

$$
\begin{aligned}
& \frac{1}{b-a} \int_{a}^{b} f\left(x, \frac{c+d}{2}\right) d x \\
\leq & \frac{1}{b-a} \int_{a}^{b}\left[s f\left(x, \frac{s d+(2-s) c}{2}\right)+(1-s) f\left(x, \frac{(1+s) d+(1-s) c}{2}\right)\right] d x \\
\leq & \frac{1}{(b-a)(d-c)} \int_{a}^{b} \int_{c}^{d} f(x, y) d y d x .
\end{aligned}
$$


Similarly applying Hadamard's inequality for the convex mapping $f_{y}:[a, b] \rightarrow \mathbb{R}, f_{y}(u)=f(u, y)$ on $[a, b]$ for all $y \in[c, d]$, we get

$$
\begin{aligned}
& \frac{1}{d-c} \int_{c}^{d} f\left(\frac{a+b}{2}, y\right) d y \\
\leq & \frac{1}{d-c} \int_{c}^{d}\left[t f\left(\frac{t b+(2-t) a}{2}, y\right)+(1-t) f\left(\frac{(1+t) b+(1-t) a}{2}, y\right)\right] d y \\
\leq & \frac{1}{(b-a)(d-c)} \int_{a}^{b} \int_{c}^{d} f(x, y) d y d x .
\end{aligned}
$$

Summing the inequalities (2.5) and (2.6), we get the second and the third inequalities in (2.4). Again by convexity of the mapping $f_{x}:[c, d] \rightarrow \mathbb{R}, f_{x}(v)=f(x, v)$ on $[c, d]$ for all $x \in[a, b]$ and using the right double inequality in (1.4) one has

$$
\frac{1}{d-c} \int_{c}^{d} f(x, y) d y \leq \frac{1}{2}[f(x, s d+(1-s) c)+s f(x, c)+(1-s) f(x, c)] \leq \frac{f(x, c)+f(x, d)}{2}
$$

and integrating this over the interval $[a, b]$, we have

$$
\begin{aligned}
& \frac{1}{(b-a)(d-c)} \int_{a}^{b} \int_{c}^{d} f(x, y) d y d x \\
\leq & \frac{1}{2(b-a)} \int_{a}^{b}[f(x, s d+(1-s) c)+s f(x, c)+(1-s) f(x, d)] d x \\
\leq & \frac{1}{2(b-a)} \int_{a}^{b}[f(x, c)+f(x, d)] d x
\end{aligned}
$$

By a similar argument applied for the mapping $f_{y}:[a, b] \rightarrow \mathbb{R}, f_{y}(u)=f(u, y)$ is convex on $[a, b]$ for all $y \in[a, b]$, we get

$$
\begin{aligned}
& \frac{1}{(b-a)(d-c)} \int_{a}^{b} \int_{c}^{d} f(x, y) d y d x \\
\leq & \frac{1}{2(d-c)} \int_{c}^{d}[f(t b+(1-t) a, y)+t f(a, y)+(1-t) f(a, y)] d y \\
\leq & \frac{1}{2(d-c)} \int_{c}^{d}[f(a, y)+f(b, y)] d y .
\end{aligned}
$$

Adding the inequalities (2.7) and (2.8), we get the fourth and fifth inequalities in (2.4) and hence completing the proof.

Corollary 2.1. Under the assumptions of Theorem 2.2, we have the following inequalities:

$$
\begin{aligned}
f\left(\frac{a+b}{2}, \frac{c+d}{2}\right) & \leq \sup _{t, s \in[0,1]} \gamma(t, s) \\
& \leq \frac{1}{(b-a)(d-c)} \int_{a}^{b} \int_{c}^{d} f(x, y) d y d x \\
& \leq \inf _{t, s \in[0,1]} \Gamma(t, s) \\
& \leq \frac{f(a, c)+f(a, d)+f(b, c)+f(b, d)}{4}
\end{aligned}
$$

where $\gamma(t, s)$ and $\Gamma(t, s)$ are as defined in Theorem 2.2. 
Corollary 2.2. With notations above, we have the following inequalities:

$$
\begin{aligned}
f\left(\frac{a+b}{2}, \frac{c+d}{2}\right) & \leq \max \left\{\sup _{0 \leq t, s \leq 1} \gamma(t, s), \sup _{0 \leq t, s \leq 1} \Omega_{2}(t, s)\right\} \\
& \leq \frac{1}{(b-a)(d-c)} \int_{a}^{b} \int_{c}^{d} f(x, y) d y d x \\
& \leq \min \left\{\inf _{0 \leq t, s \leq 1} \Gamma(t, s), B\right\} \\
& \leq \frac{f(a, c)+f(a, d)+f(b, c)+f(b, d)}{4} .
\end{aligned}
$$

Where $B, \gamma(t, s)$ and $\Gamma(t, s)$ are as defined above.

Now, we present the importance of the refinement (2.4) with the help of example.

Example 2.1. Let $\Delta=[0,1] \times[0,1]$ and $f(x, y)=x^{3} y^{3}$. Clearly the function $f(x, y)=x^{3} y^{3}$ is convex on the co-ordinates on $\Delta$.

Now,

$$
\begin{aligned}
\int_{0}^{1} \int_{0}^{1} f(x, y) d y d x & =\frac{1}{16}, \\
\mathrm{~A} & =\frac{1}{2}\left[\int_{0}^{1} f\left(x, \frac{1}{2}\right) d x+\int_{0}^{1} f\left(\frac{1}{2}, y\right) d y\right]=\frac{1}{32} \\
\Omega_{2}\left(\frac{1}{2}, \frac{1}{2}\right) & =\frac{1}{2}\left[\int_{0}^{1} \int_{0}^{1}\left[f\left(x, \frac{2 y+1}{4}\right)+f\left(\frac{2 x+1}{4}, y\right)\right] d x d y\right]=\frac{5}{128}, \\
\gamma\left(\frac{1}{2}, \frac{1}{2}\right) & =\frac{1}{4}\left[\int_{0}^{1}\left[f\left(x, \frac{1}{4}\right)+f\left(x, \frac{3}{4}\right)\right] d x+\int_{0}^{1}\left[f\left(\frac{1}{4}, y\right)+f\left(\frac{3}{4}, y\right)\right] d y\right] \\
\Gamma\left(\frac{1}{2}, \frac{1}{2}\right) & =\frac{56}{1024}, \\
& =\frac{5}{4}\left[\int_{0}^{1}\left[f\left(x, \frac{1}{2}\right)+\frac{1}{2} f(x, 1)\right] d x+\int_{0}^{1}\left[f\left(\frac{1}{2}, y\right)+\frac{1}{2} f(1, y)\right] d y\right] \\
\mathrm{B} & =\frac{1}{2}\left[\int_{0}^{1}[f(x, 0)+f(x, 1)] d x+\int_{0}^{1}[f(0, y)+f(1, y)] d y\right] \\
& =\frac{1}{8} .
\end{aligned}
$$

Therefore,

$$
\begin{aligned}
f\left(\frac{1}{2}, \frac{1}{2}\right) & =\frac{1}{64} \\
& \leq \mathrm{A}=\frac{1}{32} \\
& \leq \gamma\left(\frac{1}{2}, \frac{1}{2}\right)=\frac{56}{1024} \\
& \leq \sup _{0 \leq t, s \leq 1} \gamma(t, s) \\
& \leq \int_{0}^{1} \int_{0}^{1} f(x, y) d y d x=\frac{1}{16}
\end{aligned}
$$




$$
\begin{aligned}
& \leq \inf _{0 \leq t, s \leq 1} \Gamma(t, s) \\
& \leq \Gamma\left(\frac{1}{2}, \frac{1}{2}\right)=\frac{5}{64} \\
& \leq \mathrm{B}=\frac{1}{8} \\
& \leq \frac{f(0,0)+f(0,1)+f(1,0)+f(1,1)}{4}=\frac{1}{4},
\end{aligned}
$$

we get an estimation better than the estimation obtained in [3, Example 1] as well as in Theorem 1.3.

\section{Acknowledgements}

All the authors would like to thank the anonymous referees for their valuable reviews and comments, which improved the quality and standard of the manuscript.

\section{References}

[1] Alomari, M. and Darus, M., The Hadamard's inequality for s-convex functions of 2-variables on the co-ordinates, Int. J. Math. Anal., 2(2008), 629-638.

[2] Alamori, M. and Darus, M., On the Hadamard's inequality for log-convex functions on the coordinates, $J$. Inequal. Appl., 2009, (2009): 283147.

[3] Chen, F., A note on the Hermite-Hadamard inequality for convex functions on the co-ordinates, J. Math. Inequal., 8, 4(2014), 915-923.

[4] Chu, Y. M., Khan, M. A., Ali, T. and Dragomir, S. S., Inequalities for $\alpha$-fractional differentiable functions, J. Ineq. Appl., 2017, (2017), 1-12.

[5] Chu, Y. M., Khan, M. A., Khan, T. U. and T. Ali, Generalizations of Hermite-Hadamard type inequalities for MT-convex functions, J. Nonlinear Sci. Appl., 9, (2016), 4305-4316.

[6] Dragomir, S. S., Two Mappings in Connection to Hadamard's Inequalities, J. Math. Anal, Appl., 167, (1992), $49-56$.

[7] Dragomir, S. S., On the Hadamard's inequality for convex function on the co-ordinates in a rectangle from the plane, Taiwanese J. Math., 5, 4(2001), 775-788.

[8] Dragomir, S. S., Hermite-Hadamard's type inequalities for operator convex functions, Appl. Math. Comput., 218, 3(2011), 766-772.

[9] Dragomir, S. S., Hermite-Hadamard's type inequalities for convex functions of selfadjoint operators in Hilbert spaces, Linear Algebra Appl., 436, 5(2012), 1503-1515.

[10] Farissi, A. E., Simple proof and refinement of Hermite-Hadamard inequality, J. Math. Inequal., 4, 3(2010), 365-369.

[11] Hadamard, J., Étude sur les propriétés des fonctions entières et en particulier dune fonction considérée par Riemann, J. Math. Pures Appl., 58, (1893), 171-215.

[12] Gao, X., A note on the Hermite-Hadamard inequality, J. Math. Inequal., 4, 4(2010), 587-591.

[13] Bessenyei, M. and Páles, Z., Hadamard-type inequalities for generalized convex functions, Math. Inequal. Appl., 6, 3(2003), 379-392.

[14] Iqbal, M., Bhatti, M. I. and Nazeer, K., Generalization of inequalities analogous to Hermite-Hadamard inequality via fractional integrals, Bulletin of the Korean Math. Soc. 52(3) (2015), 707-716.

[15] Khan, M. A., Ali, T., Dragomir, S. S. and Sarikaya, M. Z., Hermite-Hadamard type inequalities for conformable fractional integrals, Revista de la Real Academia de Ciencias Exactas, Fisicas y Naturales, 2017, 2017, 1-16. 
[16] Latif, M. A. and Alomari, M., On Hadamard-type inequalities for h-convex functions on the coordinates, Int. J.Math. Anal., 3, 33(2009), 1645-1656.

[17] Latif, M. A., and Dragomir, S. S., On some new inequalities for differentiable co-ordinated convex functions,J. Inequal. Appl., 2012, (2012): 28.

[18] Nozdemir, M. E., Kavurmaci, H., Akdemir, A. O. and Avci, M., Inequalities for convex and s-convex functions on $\Delta=[a, b] \times[c, d]$, J. Inequal. Appl., 2012, (2012): 20 .

\section{Affiliations}

TAHIR ALI

AdDress: Department of Mathematics, University of Peshawar, Peshawar 25000, Pakistan.

E-MAIL: atahir623@gmail.com

ORCID ID: $0000-0003-4423-7424$

MUHAMMAD ADIL KHAN

AdDRESS: Department of Mathematics, University of Peshawar, Peshawar 25000, Pakistan.

E-MAIL: adilswati@gmail.com

ORCID ID: $0000-0001-5373-4663$

ADEM KILICMAN

ADDRESS: Department of Mathematics and Institute of Mathematical Research Universiti Putra Malaysia (UPM), 43400 UPM, Serdang, Selangor, Malaysia.

E-MAIL: akilicman@putra.upm.edu.my

ORCID ID: $0000-0002-1217-963 X$

QAMAR DIN

AdDRESS: Department of Mathematics, University of Poonch Rawalakot, Pakistan.

E-MAIL: qamar.sms@gmail.com

ORCID ID: $0000-0002-0999-7404$ 